\title{
Análisis
}

\section{Web móvil y su implantación en la administración local española}

\author{
Por Ramón Voces-Merayo y Lluís Codina
}

\begin{abstract}
Resumen: En los últimos años las tecnologías móviles han ido incrementando su importancia en el ecosistema informativo social y hoy en día se han convertido en una opción real de acceso a la Web. Cada vez hay más personas que utilizan la web móvil y cada vez aparecen más portales que ofrecen servicios para este tipo de usuarios. Se introduce el concepto de web móvil y se esbozan sus características principales para después ofrecer una radiografía del impacto que está produciendo en la administración local española, tanto desde el punto de vista cuantitativo como cualitativo.

Palabras clave: Web móvil, Administración local, España, mobileOK, Xhtml-MP, WCSS

Title: Mobile web and its introduction in the Spanish local administration

Abstract: In recent years, mobile technologies have become more and more important in the social information ecosystem and they are a real option for accessing the Web. Each time there are more and more people that use the mobile web, and each time there are more portals that offer services for this kind of users. The web mobile concept and its main characteristics are presented, as well as the degree of adoption of the mobile web in the local Spanish administration, both quantitatively and qualitatively.

Keywords: Mobile web, Local administration, Spanish, MobileOK, Xhtml-MP, WCSS

Voces-Merayo, Ramón; Codina, Lluís. "Web móvil y su im-

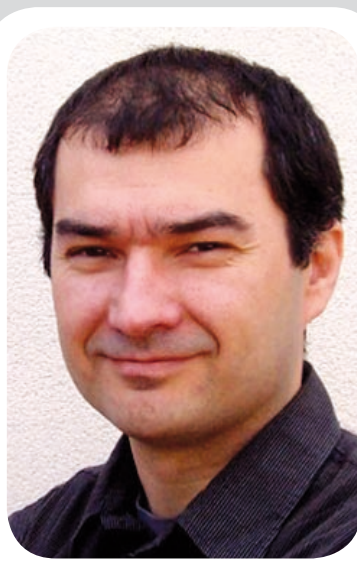

Ramón Voces-Merayo, ingeniero técnico en telecomunicaciones, licenciado en documentación y diploma en estudios avanzados por la Univ. Autónoma de Barcelona, es profesor asociado en la UAB donde imparte docencia en los estudios de documentación-, y colaborador de la UOC. Es miembro del Grupo de Invest. Laicom de la UAB y participa en el grupo DigiDoc de la UPF. Sus intereses se centran en las tecnologías de la web, sobre todo publicación digital, multimedia y accesibilidad, temas de su futura tesis doctoral.

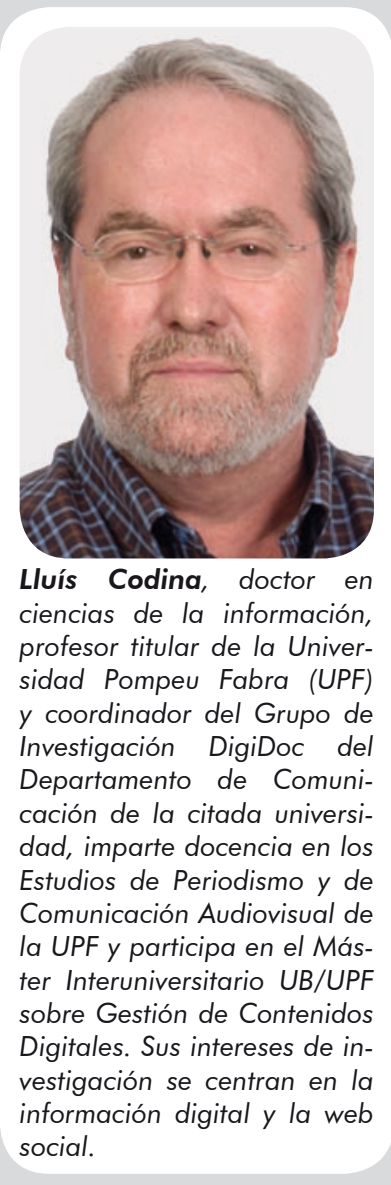
plantación en la administración local española”. El profesional de la información, 2009, marzo-abril, v. 18, n. 2, pp. 211217.

DOI: 10.3145/epi.2009.mar.11
\end{abstract}

\section{Introducción}

NO ES DIFÍCIL ENCONTRAR PREDICCIONES sobre el futuro de las comunicaciones móviles. Independientemente de la fuente consultada todos los análisis parecen llegar a una conclusión parecida: el mercado de la telefonía móvil tendrá una penetración cada vez mayor y el uso de la, así llamada, web móvil será cada vez más habitual.

Seguramente por esta razón no es sorprendente, a pesar de la magnitud, leer que existen alrededor de 4.000 millones de líneas móviles activadas en todo el mundo (ITU, 2008); o que en países como Japón hay más líneas móviles que fijas y que un $82,7 \%$ de la población entre 6 y 79 años se conecte a la web móvil (Ministry of Internal Affairs and Communications, 2008); o que en España más de 30 millones de personas dispongan de un teléfono móvil y que el porcentaje de usua- rios que accede a la web móvil se vaya incrementando año tras año'; $o$, finalmente, que haya estudios que profeticen que los móviles llegarán en breve a ser rivales de los PCs como plataformas de acceso a internet (Ipsos, 2006).

Pero probablemente lo más significativo es que estos avances no sólo se quedan en los países desarrollados. Por poner un ejemplo, en Uganda en el año 2008 sólo el 0,5\% de la población disponía de una lí- 
nea de telefonía fija, frente al $21 \%$ que tenía una línea móvil (con un incremento del $69 \%$ respecto al año anterior) lo que permitía a cerca del $10 \%$ de sus habitantes tener acceso a internet (Uganda Communications Commission, 2008). Sin duda, una posibilidad real para reducir la división digital.

Se mire por donde se mire los avances en la telefonía móvil están cambiando drásticamente el panorama de las comunicaciones en el mundo: el hecho de poder conectarse de forma fácil, instantánea, allá donde estés..., esto es, tener la posibilidad de llevar la Web en el bolsillo, ofrece un enorme conjunto de posibilidades de las que, sin duda, seremos espectadores en los próximos años.

Este trabajo se estructura en dos partes: en la primera se examina el concepto de web móvil y se presentan los elementos clave que involucra para facilitar su comprensión. En la segunda parte se analiza su grado de implantación y la calidad de la misma en el caso de la administración local española, utilizando una herramienta de evaluación automática que nos ha permitido establecer un primer mapa sencillo de la cuestión. Finalmente, presentamos unas conclusiones y unas posibles líneas futuras de trabajo para esta nueva web que, sin duda, ocupará una parte cada vez más importante de nuestro futuro profesional.

\section{La web móvil}

La importancia de la relación entre la web y las comunicaciones móviles es tal que ya se ha acuñado el término mobile web (web móvil). En principio, entendemos por web móvil el acceso a la World Wide Web utilizando dispositivos móviles.

Ahora bien, para comprender la web móvil en su contexto es necesario analizar los elementos que intervienen. De una forma muy esquemática se podrían considerar que son los 4 siguientes: tecnolo- gías, usuarios, desarrollos y contenidos.

\subsection{Tecnologías}

El principal organismo internacional encargado de elaborar especificaciones tecnológicas para móviles es la Open Mobile Alliance (OMA).

\section{http://www.openmobilealliance.} org/

La $O M A$ es un consorcio creado en 2002 que agrupa los principales representantes de las tecnologías móviles (desde fabricantes de dispositivos hasta operadoras de telefonía, pasando por un gran número de compañías de software) y que tiene por objetivo ayudar en el desarrollo de sistemas de comunicación móviles interoperables entre países, operadores y terminales.

En lo que se refiere a internet, OMA es responsable del Wireless application protocol (WAP), un estándar que define los protocolos y los lenguajes de marcas que permiten el acceso a internet utilizando dispositivos móviles.

En su última versión, la WAP 2.0, se ha superado un gran número de dificultades que presentaba la versión anterior, la WAP 1.0, y se ha avanzado en la línea de una mayor integración con los protocolos y los lenguajes de la web "estándar". En este sentido, probablemente, los avances más significativos se dan en estos tres apartados:

- Protocolos internet. Se añaden protocolos compatibles con los tcp/ip. De esta manera el acceso a internet se hace directamente sin necesidad de pasar, como en la versión anterior, por una pasarela WAP (WAP gateway).

- Lenguaje de marcas. Se deja atrás el lenguaje de marcas WML (wireless markup language) de WAP 1.0 y se crea uno nuevo denominado xhtml-MP (Mobile profile), basado en la recomendación Xhtml basic del W3C. De esta forma ya no es necesario aprender un nuevo lenguaje de marcas para realizar páginas web para móviles.

- Hojas de estilo WCSS (Wap CSS). Se mejora la presentación de la información con la posibilidad de añadir hojas de estilo en cascada, tomando como base la recomendación CSS2 Specification del W3C.

Además, WAP 2.0 se concibe como una extensión de WAP 1.0, por lo que se puede decir que WAP 2.0 integra WAP 1.0 y de esta forma se asegura la compatibilidad con los dispositivos más antiguos.

Por otro lado, también es necesario destacar las actividades del W3C. Más allá de las evidentes influencias que aporta a los trabajos de la $O M A$, en 2005 se creó una nueva iniciativa denominada Mobile web initiative (MWI) con el objetivo de mejorar la interoperatibilidad y la usabilidad en los entornos móviles, que actualmente está compuesta por cuatro grupos de trabajo. Como veremos más adelante, algunos de sus resultados ya han llegado al estado de recomendación del $W 3 C \mathrm{y}$ se han convertido en un marco de referencia para los desarrolladores.

\subsection{Usuarios}

En muchas ocasiones el éxito o fracaso de un sitio web depende del conocimiento que se tiene de los usuarios a los que se dirige (usuarios-objetivo). Un conocimiento detallado de la tipología de usuarios y de sus respectivos contextos ayuda a ofrecer los servicios que requieren en las condiciones que los necesitan y, en consecuencia, a implementar una sede web útil, usable y accesible.

En este sentido, para el desarrollo de una web móvil es necesario responder a una serie de cuestiones sobre el perfil genérico de sus usuarios. Seguramente las más importantes son estas tres:

- ¿Desde qué lugar acceden los usuarios a la web móvil? 
- ¿Cómo la utilizan?

- ¿Con qué tipo de dispositivos acceden?

En lo que respecta al primer punto, y a pesar de que inicialmente sería lógico pensar que la web móvil la utilizan sobre todo los usuarios que están en tránsito, lo cierto es que su uso es muy común desde lugares fijos (como el hogar o la oficina). Esto se debe a que la inmediatez y la facilidad del acceso es mucho mayor que desde el ordenador de escritorio (ya que el dispositivo móvil siempre suele estar conectado y muy cercano al usuario) y al hecho de que se pueda acceder sin necesidad de interrumpir la actividad principal que se esté haciendo (Mehta, 2008).

\section{"El acceso online es más fácil que desde el PC ya que el móvil siempre suele estar a mano y conectado"}

En lo que se refiere a cómo se utiliza la web móvil, y dadas sus características, cabe destacar dos tipos fundamentales de uso:

- Informativo. Responde al usuario que tiene una necesidad de información muy puntual y muy definida (como encontrar una dirección, un número de teléfono, unos horarios o confirmar una reserva). Pueden ser necesidades que requieran resolverse rápidamente puesto que podrían ser esenciales para comenzar una nueva tarea.

- Lúdico. Es el del usuario que se conecta en los tiempos muertos entre dos actividades. Por ejemplo, mientras espera en un restaurante o durante los espacios publicitarios mientras ve la televisión en casa. En estos casos el acceso a la web se hace sin ningún tipo de objetivo definido.
Finalmente, también es importante conocer con qué tipo de dispositivos se accede, de los que existe una gran variedad. No sólo se diferencian en su diseño y usabilidad sino también en las características tanto físicas: área de visualización, peso, memoria, etc., como lógicas: aplicaciones disponibles o el tipo y la versión del micronavegador (el navegador específico para la web móvil) $)^{2}$.

Básicamente existen tres tipos de dispositivos móviles:

1) Móvil convencional. Es el segmento más básico. Normalmente poseen un total de 12 teclas y disponen de servicio de voz, mensajería y datos. Además pueden tener otras características adicionales como cámaras de vídeo o reproductores multimedia.

2) Teléfonos inteligentes o smart phones. Son un tipo de dispositivos con los que se pueden ejecutar funciones avanzadas (acceso a correo electrónico, agenda...). Disponen de un sistema operativo completo que permite instalar nuevas aplicaciones, así como, algunos, de un teclado alfabético. Suelen tener un área de visualización (pantalla) mayor que los teléfonos móviles convencionales.

3) PDA. Inicialmente diseñados como agenda electrónica (personal digital assistant) en los últimos años han evolucionado hasta ser un verdadero ordenador de mano con las posibilidades de conexión de un teléfono móvil. La pantalla puede ser mayor que la de un smart pho$n e$, ya que el uso primario suele ser como organizador personal ${ }^{3}$.

El más común de los tres tipos es el móvil convencional (Cremin et al., 2007), pero en cualquier caso es importante remarcar que, independientemente del dispositivo elegido, el usuario se encuentra con un buen número de limitaciones que no existen en otros entornos:

1. El área de visualización es reducida o muy reducida.
2. En la mayoría de los casos no se dispone de ningún lápiz o sistema apuntador y el teclado suele ser limitado y con las teclas pequeñas.

3. Limitaciones de memoria y funcionales como, por ejemplo, de ejecución de scripts.

4. La velocidad de conexión es mucho menor.

5. En función del tipo de conexión suelen incurrir en un coste asociado, que generalmente depende del volumen de datos y que puede llegar a ser elevado.

\subsection{Desarrollos}

Desde el punto de vista de producción, la web móvil presenta aspectos positivos y aspectos negativos.

Uno claramente positivo es tecnológico: el hecho de que el lenguaje sea xhtml-MP y WCSS facilita en gran medida el trabajo del desarrollador, que ya no tiene necesidad de aprender otros lenguajes (como ocurría con el WML de WAP 1.0). Además, hace posible trabajar con las aplicaciones tradicionales usadas en la creación de sedes web de escritorio convencionales.

El aspecto negativo radica en el dispositivo: por un lado, y consecuencia de las limitaciones físicas y lógicas ya mencionadas, las características de la estructura de la página web y del contenido que presenta son diferentes a la web "estándar" o de escritorio, como la denominaremos a partir de ahora.

Por otro lado, existen múltiples versiones de micronavegadores, cada uno de ellos con decenas de variantes, con lo que se hace difícil conseguir una visualización correcta que sea independiente del micronavegador $\mathrm{y}$, a la vez, aprovechar al máximo las características del aparato con el que se conecta.

Como resultado de las consideraciones anteriores, los desarrolladores se enfrentan a dos cuestiones importantes: 
1. ¿Cuál es la relación entre la web de escritorio y la web móvil?

2. ¿Cómo se puede asegurar que el contenido que se está preparando pueda ser visto en cualquier dispositivo con micronavegador?

Para la primera cuestión, y a pesar de que el $W 3 C$ considera una buena práctica disponer de una única sede web que sea capaz de adaptarse a las características de cada dispositivo ( $W 3 C, 2008$ ), lo cierto es que la gran mayoría de desarrolladores entiende que el entorno móvil presenta unas características suficientemente diferenciadas como para justificar un diseño específico y paralelo a la sede web utilizada para los usuarios de escritorio.

De esta manera, es muy frecuente observar cómo el acceso a la web móvil se hace mediante URLs específicos, ya sea mediante la creación de un dominio específico (el .mobi, como http://empresa. mobi) o mediante la creación de un subdominio (como http://movil.empresa.com) o de una carpeta dentro de la sede web de escritorio (como http://www.empresa.com/movil).

Evidentemente, en estos casos el reto es conseguir que la información proporcionada desde ambas sedes sea siempre coherente.

Respecto a la segunda cuestión, el $W 3 C$ ha publicado dos herramientas fundamentales para los desarrolladores:

1. El Default delivery context (DDC) que establece las características mínimas que debe tener un dispositivo móvil para poder disfrutar de una experiencia de usuario razonable.

2. La recomendación mobileOK Basic Test 1.0, que consiste en una serie de tests pensados para ejecutarse de forma automatizada, para comprobar si un contenido puede visualizarse adecuadamente en un dispositivo que cumple con las especificaciones DDC. En el caso de superarlos, la página o sede web puede marcarse con el sello mobileOK del $\mathrm{W}^{3} \mathrm{C}^{4}$.

Desde el punto de vista del realizador, el DDC es un modelo genérico sobre el que basar las producciones y el mobileOK una forma de evaluar su funcionamiento básico.

Sin embargo, el DDC es un compromiso de mínimos y se entiende que el realizador debe esforzarse por conseguir siempre la mejor experiencia de usuario posible. Y esto implica identificar las características del móvil y mostrar un contenido que se adapte a las mismas.

Lo más conveniente es disponer de ejemplares de los equipos (del móvil, de la PDA, etc.) para los que se elabora la página. Si esto no es posible, lo más habitual es trabajar con emuladores, aunque a veces pueden presentar diferencias significativas respecto al dispositivo real.

Existen diversas formas de conseguir los emuladores, bien sea a través del mismo fabricante, que vengan integrados en paquetes de producción, o bien adquiriéndolos a terceras empresas.

\subsection{Contenidos}

Las características del usuario de la web móvil y del dispositivo con el que se conecta condicionan en gran medida tanto el contenido como la estructura de la página web.

En general, a la hora de diseñar el contenido se considera prioritaria la persona que hace un uso puntual (informativo). Por lo tanto, los objetivos del diseño deben ir enfocados a conseguir que el usuario acceda de la forma más rápida posible a la información que precisa (W3C, 2008).

De todos modos ya hemos mencionado que también hay usuarios que hacen un uso lúdico de la web móvil, de forma que se considera una buena práctica permitir que se pueda decidir qué tipo de experiencia desea: si la expresamente diseñada para la web móvil o una versión más completa.

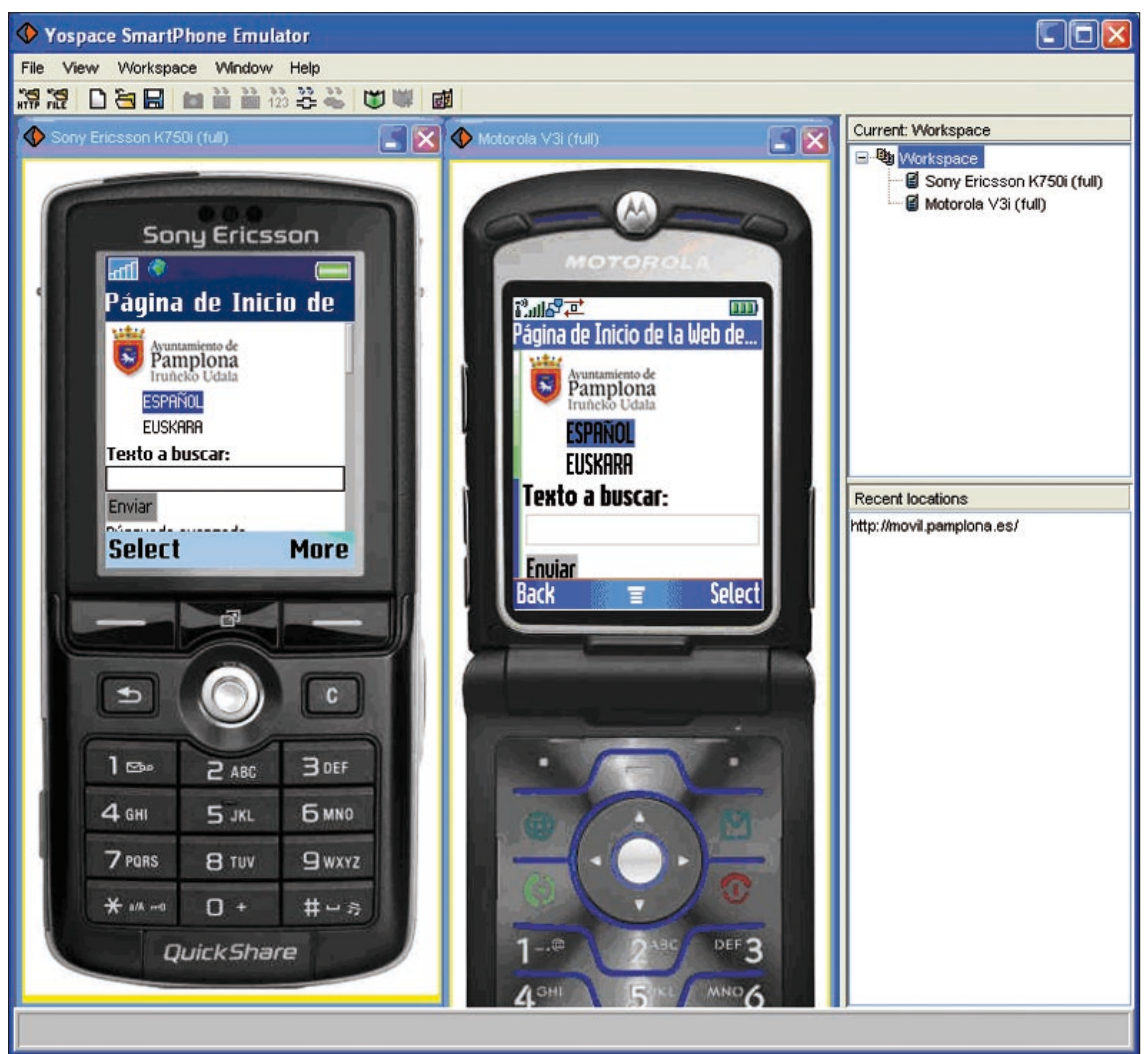

Figura 1. Aplicación Yospace Smartphone Emulator 
En todo lo que se refiere a buenas prácticas, seguramente el documento más relevante es el $\mathrm{Mo}$ bile web best practices 1.0, una recomendación del $W 3 C$ de julio de 2008, en la que se detalla un conjunto de 60 buenas prácticas que engloban desde aspectos generales, hasta consideraciones respecto a la estructura de la página, la navegación o el diseño del contenido.

\section{"Una buena política de información otorga prestigio y legitimidad a una administración pública"}

\section{La web móvil en la administración local española}

Sin duda alguna la web móvil representa un terreno lleno de oportunidades. Desde el punto de vista de la administración local puede ser una herramienta fundamental para incrementar el valor de la sede web municipal y, al mismo tiempo, una ocasión para establecer un nuevo canal de comunicación con los ciudadanos. Como es sabido, las buenas políticas de información son, cada vez más, políticas que otorgan a la vez prestigio y legitimidad a las administraciones que hacen un buen uso de ellas.

En el caso de la web móvil, las preguntas que nos hemos planteado para este estudio son dos:

1. ¿Cuál es el grado de implantación de la web móvil en la administración local?

2. ¿Cómo se está desarrollando? O dicho de otra forma: ¿se está desarrollando con la calidad suficiente como para asegurar que van a ser sitios funcionales para los ciudadanos?

\subsection{Método}

Para hacer este estudio se han analizado las 50 sedes web de los ayuntamientos de todas las capitales de provincia de España en el periodo comprendido entre el 20 de enero de 2009 y el 9 de febrero de 2009, teniéndose en cuenta los siguientes elementos:

- Disponibilidad de portal móvil. Se analiza la página principal de la sede web de escritorio del ayuntamiento en busca de un acceso directo a la web móvil. En el caso de no encontrarse se estudia el mapa de la web (si existe) y en última instancia se utiliza el sistema de búsqueda propio de la web (si existe) mediante las palabras clave: móvil (con las variantes autonómicas correspondientes) y PDA.

- Calidad de la implantación. Se analiza la página principal con un evaluador automático disponible en

http://ready.mobi

En este caso es preciso hacer notar que:

a. Se ha optado por utilizar el evaluador

http://ready.mobi

en lugar del propio del W3C

http://validator.w3.org/mobile/

por dos razones:

i. El evaluador del W3C todavía presenta algunos problemas de implementación.

ii. El de ready.mobi incluye la gran mayoría de los tests de mobile $O K$ y proporciona un sistema de puntuación que permite establecer comparaciones entre las sedes web evaluadas.

b. El resultado de esta evaluación es numérico, entre 0 y 5.

c. Se evalúa únicamente si el contenido presenta elementos básicos de usabilidad, eficiencia e interoperabilidad. En ningún caso se evalúa si es el adecuado para móviles ni si son útiles para sus usuarios.

\subsection{Resultados}

\section{- Implantación}

Como se puede observar en la tabla 1, tan sólo 10 ayuntamientos (Cuenca, Lleida, Logroño, Castellón de la Plana, Almería, San Sebastián, Pamplona, Zaragoza, Barcelona y Madrid) de los 50 analizados tienen un portal móvil específico, lo que representa una implantación del $20 \%$.

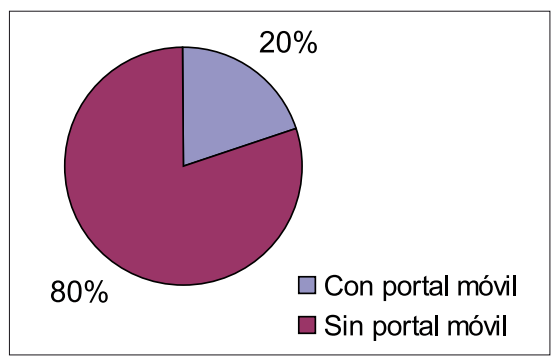

Figura 2. España: capitales de provincia y web móvil

A pesar de que este número es muy pequeño, no hay que olvidar que la mayoría los portales móviles aparecen en las capitales más pobladas, y por lo tanto el número de ciudadanos con acceso a un portal móvil es considerablemente mayor de lo que este exiguo porcentaje da a entender inicialmente.

En concreto, las 50 capitales analizadas suman 13.782.693 habitantes; de ellas, las 10 capitales que disponen de portal móvil representan 6.025.446, lo que implica un $43,7 \%$ del conjunto de la población estudiada.

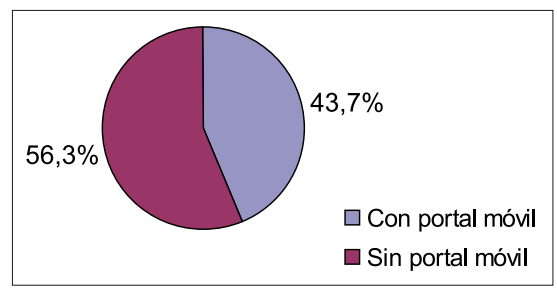

Figura 3. España: habitantes y web móvil

\section{- Calidad de la implantación}

En lo que respecta a los parámetros de calidad, se obtiene una nota media de 2,8 , nota que si la ponde- 


\begin{tabular}{|r|l|c|l|c|}
\hline No & \multicolumn{1}{|c|}{ Capital } & Habitantes $^{\mathbf{1}}$ & \multicolumn{1}{|c|}{ Portal móvil $^{-}$} & Calidad $^{\mathbf{6}}$ \\
\hline 4 & Cuenca & 46.341 & http://www.cuenca.es/movil & 3,6 \\
\hline 17 & Lleida & 112.199 & http://lleida.mobi & 3,5 \\
\hline 21 & Logroño & 133.058 & http://www.logro-o.org/pda/ & 2,4 \\
\hline 25 & $\begin{array}{l}\text { Castellón de } \\
\text { la Plana }\end{array}$ & 147.667 & http://www.castello.es/wap/ & 2,3 \\
\hline 29 & Almería & 166.328 & http://www.aytoalmeria.es/ciudadano/pda/nav/index.jsp & 1,3 \\
\hline 30 & San Sebastián & 178.377 & http://movil.donostia.org/ & 1,0 \\
\hline 32 & Pamplona & 183.964 & http://movil.pamplona.es/ & 2,4 \\
\hline 46 & Zaragoza & 614.905 & http://www.zaragoza.mobi & 3,2 \\
\hline 49 & Barcelona & 1.503 .884 & http://barcelona.mobi & 3,7 \\
\hline 50 & Madrid & 2.938 .723 & $\begin{array}{l}\text { http://www.munimadrid.es/portal/site/munimadrid/menuitem.80293a } \\
\text { 1aad00ed52a1bf17d19fc08a0c/?vgnextoid=1ccd566813946010Vgn } \\
\text { VCM100000dc0ca8c0RCRD\&vgnextfmt=pda }\end{array}$ & 4,9 \\
\hline
\end{tabular}

Tabla 1. Capitales de provincia españolas que disponen de web móvil ciudadana, ordenadas por número de habitantes

ramos con respecto a la población a la que afecta se incrementa hasta los 4,0 puntos (por el mayor peso que aportan Madrid y Barcelona).

A pesar de que en primera instancia se puede decir que el resultado es positivo, no hay que olvidar que este test evalúa aspectos muy básicos. En la mayoría de los casos sería relativamente fácil mejorar la puntuación realizando pequeños cambios en la implementación.

Es fácil detectar en la tabla 1 y en la figura 4 que existen resultados muy dispares si comparamos capitales como San Sebastián y Almería con puntuaciones de 1 o poco más con Madrid que se acerca a 5. En todo caso, el promedio de calidad es insuficiente si apartamos el caso de Madrid.

Las siguientes imágenes son capturas de ejemplo de algunos

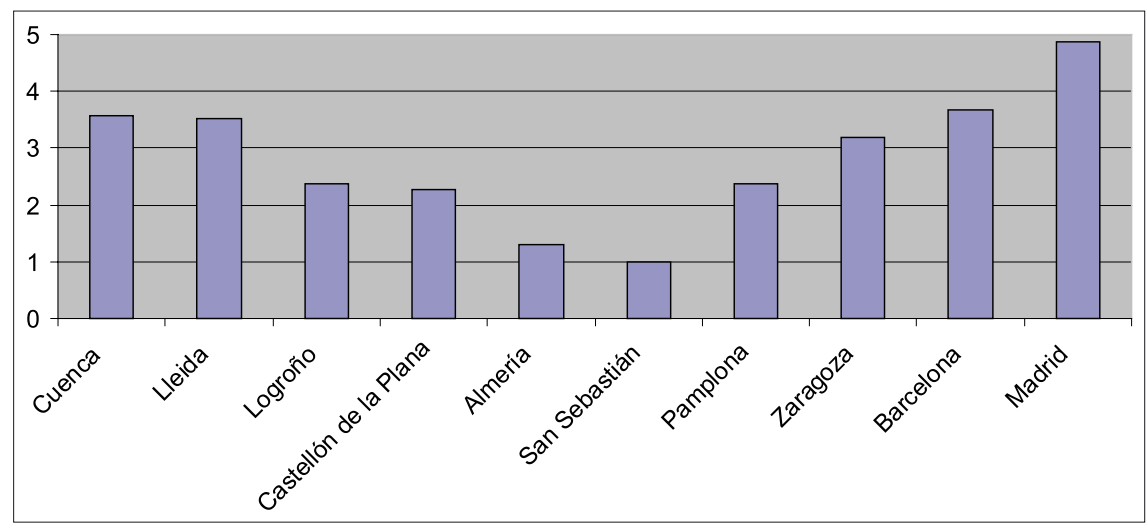

Figura 4. Capitales y calidad de la web móvil

de los servicios de web móvil comentados en este trabajo, y se han realizado utilizando un simulador Openwave v7.

\section{Conclusiones}

4.1. Escasa implantación. Un total de 10 ayuntamientos de capitales de provincia españoles habían dado pasos a inicios de 2009 en re-

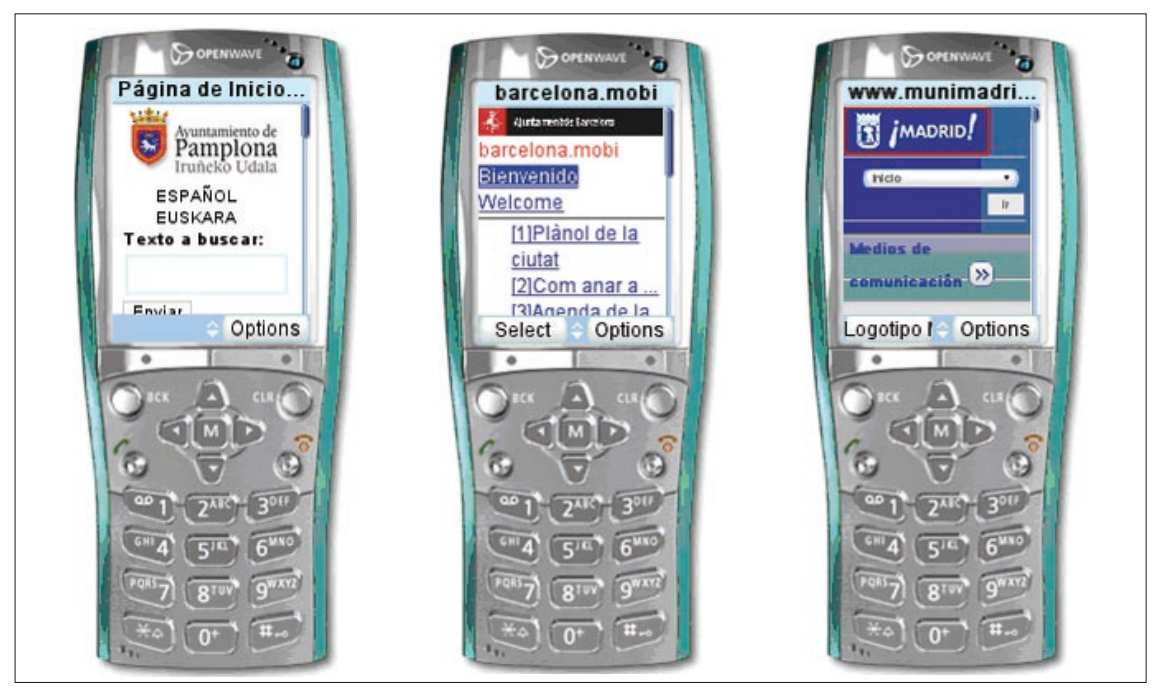

Figura 5. Portales móviles de Pamplona, Barcelona y Madrid lación con la web móvil, sin duda, la web del futuro.

Es destacable el papel de los ayuntamientos de Cuenca, Lleida, Logroño, Castellón de la Plana, Almería, San Sebastián y Pamplona, ciudades relativamente pequeñas $\mathrm{y}$ que cuentan ya con portales móviles.

Aunque por porcentaje de población representa casi un $44 \%$ del total, no deja de ser una cuestión de ver el vaso medio lleno o medio vacío. Dada la importancia y la enorme calidad de vida que aportan los sistemas de información a los ciudadanos, creemos que hay que ver el vaso medio vacío y reclamar de las administraciones que aún no lo hayan hecho, que incorporen esta cuestión a su cartera de asuntos pendientes.

4.2. Calidad mejorable. De los diez casos considerados, hemos podido ver que solamente Madrid alcanzaba valores altos de calidad según el test aplicado. La cuestión es que, 
como nota media se obtiene un 2,8 sobre 5, y un 4 si se realiza una media ponderada respecto de la población.

En este apartado ya hemos destacado el papel del Ayuntamiento de Madrid que no ha conseguido la puntuación máxima porque el volumen de su página principal excedía (por muy poco) el recomendado en el mobileOK Basic tests del W3C.

En general sería interesante que los ayuntamientos hicieran un esfuerzo para corregir los errores de implementación, entre otras razones porque, en muchos casos, se consiguen resolver con pequeñas modificaciones del código fuente.

\section{"La web móvil genera nuevas oportunidades de trabajo"}

\subsection{Nuevo terreno laboral} Pongamos los puntos 1 y 2 anteriores en positivo: para los profesionales de la información y la documentación se abre un nuevo camino.

Aún son relativamente pocos los profesionales que conocen bien las características de la web móvil y, sin embargo, tanto la Administración como las empresas (sobre todo medianas y grandes) van a necesitar trabajadores cualificados en esta nueva web.

Los que ya conozcan la web móvil o los que empiecen pronto a entrar en este terreno van a tener una ventaja competitiva durante los próximos años.

De todos modos, quienes nos movemos en temas de la web (nunca mejor dicho) vamos a tener que entrar en estos aspectos, por lo que mejor entrar antes que después.

\section{Líneas futuras}

Este artículo nace con la intención de promover el uso de la web móvil en la administración local. A partir del mismo hay múltiples líneas de trabajo que podrían resultar altamente interesantes:

- Análisis de la evolución de la web móvil

Sería muy útil analizar periódicamente los avances de la web móvil tanto desde el punto de vista cuantitativo como cualitativo.

- Estudio avanzado de la calidad de la web móvil

Como se ha dicho anteriormente, la evaluación de la calidad en este artículo se ha realizado analizando factores puramente tecnológicos y con evaluadores automáticos. Habría que realizar un estudio en profundidad que incluyera como mínimo la utilidad, la usabilidad y la accesibilidad como factores cualitativos de la web móvil.

- Adaptabilidad de la web móvil

Seguramente como un factor relacionado con la calidad pero suficientemente autónomo como para ser evaluado de forma separada, sería muy interesante analizar cómo se adaptan las sedes web móviles a la diversidad de móviles.

- Nuevos criterios de usabilidad

La web móvil debería adoptar también criterios de calidad relacionados con la usabilidad, pero obviamente adaptados a este nuevo escenario.

\section{Notas}

1. Datos resultantes del análisis de las Encuestas sobre equipamiento y uso de tecnologías de la información y comunicación en los hogares del INE.

http://www.ine.es/jaxi/menu.do?type $=$ pcaxis \& $p$ ath $=\% 2 F t 25 / p 450 \&$ file $=$ inebase $\& L=0$

2. A pesar de las diferencias, la gran mayoría de dispositivos dispone de un menú que permite de una forma u otra introducir un URL.

3. Ciertamente, la diferencia entre una $P D A$ y un Smart Phone es cada vez más reducida y seguramente en un futuro muy cercano serán conceptos equivalentes.

4. En el momento en el que se escribe este artículo el $W 3 C$ todavía no ha definido el procedimien- to preciso de notificación del sello mobileOK.

5. Según el censo de población de 2001 elaborado por el $I N E$.

6. Puntuación sobre 5 puntos.

\section{Bibliografía}

1. Cremin, R. et al. DotMobi mobile web developer guide. $1^{\mathrm{a}}$ ed., 2007.

2. Ipsos. Mobile phones could soon rival the PC as world's dominant internet platform. http://www.ipsos-na.com/news/pressrelease. cfm?id=3049\#

3. ITU. Worldwide mobile cellular subscribers to reach 4 billion mark late 2008

http://www.itu.int/newsroom/press_releases/ 2008/29.html

4. Mehta, N. Mobile web development. $1^{\mathrm{a}}$ ed. Birmingham: Packt, 2008. ISSN 978-1-84719343-8.

5. Ministry of Internal Affairs and Communications. Communications usage trend survey in 2007 compiled.

http://www.johotsusintokei.soumu.go.jp/tsusin riyou/data/eng_tsusin_riyou02_2007.pdf

6. Uganda Communications Commission. The post \& telecommunications performance June 07

- June 08 annual review.

http://www.ucc.co.ug/EndOfFY_Review07_ 08.pdf

7. W3C. Mobile Web Best Practices 1.0.

http://www.w3.org/TR/mobile-bp/

8. W3C. W3C mobileOK basic tests 1.0. http://www.w3.org/TR/mobileOK-basic10-tests/

9. WAP Forum. WAP 2.0 technical white paper. http://www.wapforum.org/what/WAPWhite Paper1.pdf

10. WAP Forum. Xhtml mobile profile. http://www.openmobilealliance.org/tech/affiliates/ wap/wap-277-xhtmlmp-20011029-a.pdf

11. Yanqing, C.; Virpi, R. How people use the web on mobile devices. Proc. of the 17th intl. conf. on world wide web, pp. 905-914, 2008. ISSN 978-1-60558-085-2.

Ramón Voces-Merayo, Depto. de Comunicación Audiovisual, Universidad Autónoma de Barcelona, Edifici I, Campus de la UAB, 08193 Bellaterra (Barcelona), España.

Tel.: +34-935814458

ramon.voces@uab.cat

Lluís Codina, Depto. de Comunicación, Univ. Pompeu Fabra, Roc Boronat, 138, desp. 53.804, 08018 Barcelona, España.

Tel.: +34-935421311

lluis.codina@upf.edu

http://www.lluiscodina.com 